OPEN ACCESS

Edited by:

Shuihua Wang,

University of Leicester,

United Kingdom

Reviewed by:

Yi Chen,

Nanjing Normal University, China

Blaise Frederick,

Harvard Medical School,

United States

${ }^{*}$ Correspondence:

Michael Germuska

germuskam@cardiff.ac.uk

Specialty section: This article was submitted to Medicine and Public Health,

a section of the journal

Frontiers in Artificial Intelligence

Received: 31 May 2019 Accepted: 09 March 2020

Published: 31 March 2020

Citation:

Germuska M, Chandler HL, Okell T, Fasano F, Tomassini V, Murphy $K$ and Wise RG (2020) A Frequency-Domain

Machine Learning Method for

Dual-Calibrated fMRI Mapping of Oxygen Extraction Fraction (OEF) and Cerebral Metabolic Rate of Oxygen Consumption $\left(\mathrm{CMRO}_{2}\right)$.

Front. Artif. Intell. 3:12. doi: 10.3389/frai.2020.00012

\section{A Frequency-Domain Machine Learning Method for Dual-Calibrated fMRI Mapping of Oxygen Extraction Fraction (OEF) and Cerebral Metabolic Rate of Oxygen Consumption $\left(\mathrm{CMRO}_{2}\right)$}

\author{
Michael Germuska ${ }^{1 *}$, Hannah Louise Chandler ${ }^{1}$, Thomas Okell ${ }^{2}$, Fabrizio Fasano ${ }^{3}$, \\ Valentina Tomassini ${ }^{1,4,5}$, Kevin Murphy ${ }^{1}$ and Richard G. Wise ${ }^{1,5,6}$ \\ ${ }^{1}$ Cardiff University Brain Research Imaging Centre (CUBRIC), Department of Psychology, Cardiff University, Cardiff, \\ United Kingdom, ${ }^{2}$ FMRIB, Nuffield Department of Clinical Neurosciences, Wellcome Centre for Integrative Neuroimaging, \\ University of Oxford, Oxford, United Kingdom, ${ }^{3}$ Siemens Healthcare Ltd., Camberley, United Kingdom, ${ }^{4}$ Division of \\ Psychological Medicine and Clinical Neurosciences, Cardiff University School of Medicine, Cardiff, United Kingdom, \\ ${ }^{5}$ Department of Neuroscience, Imaging and Clinical Sciences, "G. D'Annunzio University" of Chieti-Pescara, Chieti, Italy, \\ ${ }^{6}$ Institute for Advanced Biomedical Technologies, "G. D'Annunzio University" of Chieti-Pescara, Chieti, Italy
}

Magnetic resonance imaging (MRI) offers the possibility to non-invasively map the brain's metabolic oxygen consumption $\left(\mathrm{CMRO}_{2}\right)$, which is essential for understanding and monitoring neural function in both health and disease. However, in depth study of oxygen metabolism with MRI has so far been hindered by the lack of robust methods. One MRI method of mapping $\mathrm{CMRO}_{2}$ is based on the simultaneous acquisition of cerebral blood flow (CBF) and blood oxygen level dependent (BOLD) weighted images during respiratory modulation of both oxygen and carbon dioxide. Although this dual-calibrated methodology has shown promise in the research setting, current analysis methods are unstable in the presence of noise and/or are computationally demanding. In this paper, we present a machine learning implementation for the multi-parametric assessment of dual-calibrated fMRI data. The proposed method aims to address the issues of stability, accuracy, and computational overhead, removing significant barriers to the investigation of oxygen metabolism with MRI. The method utilizes a time-frequency transformation of the acquired perfusion and BOLD-weighted data, from which appropriate feature vectors are selected for training of machine learning regressors. The implemented machine learning methods are chosen for their robustness to noise and their ability to map complex non-linear relationships (such as those that exist between BOLD signal weighting and blood oxygenation). An extremely randomized trees (ET) regressor is used to estimate resting blood flow and a multi-layer perceptron (MLP) is used to estimate $\mathrm{CMRO}_{2}$ and the oxygen extraction fraction (OEF). Synthetic data with additive noise are used to train the regressors, with data simulated to cover a wide range of physiologically plausible parameters. The performance of the implemented analysis method is compared to published methods both in simulation and with in-vivo data $(n=30)$. The proposed 
method is demonstrated to significantly reduce computation time, error, and proportional bias in both $\mathrm{CMRO}_{2}$ and OEF estimates. The introduction of the proposed analysis pipeline has the potential to not only increase the detectability of metabolic difference between groups of subjects, but may also allow for single subject examinations within a clinical context.

Keywords: calibrated-fMRI, oxygen extraction fraction, $\mathrm{CMRO}_{2}, \mathrm{OEF}$, machine learning

\section{INTRODUCTION}

Under normal conditions the brain's energy needs are met via a continuous supply of oxygen and glucose for the local production of ATP via aerobic metabolism (Verweij et al., 2007). Any disruption of the supply of oxygen to the brain tissue can have significant consequences (Safar, 1988), and impaired cerebral oxygen metabolism is associated with a wide variety of neurological conditions (Frackowiak et al., 1988; Ishii et al., 1996; Miles and Williams, 2008). Therefore, monitoring and mapping the brain's consumption of oxygen is vital for understanding the diseases and mechanisms by which the metabolic consumption of oxygen may be affected. The cerebral metabolic rate of oxygen consumption $\left(\mathrm{CMRO}_{2}\right)$ has traditionally been measured with positron emission tomography (Frackowiak et al., 1980). However, this method has some substantial limitations including the use of ionizing radiation and the need for local production of 15-oxygen labeled tracers. Due to these limitations there is great interest in developing alternative, non-invasive, methods of mapping $\mathrm{CMRO}_{2}$. One promising technique of non-invasively mapping $\mathrm{CMRO}_{2}$ is the so-called dual-calibrated fMRI (dcfMRI) method (Bulte et al., 2012; Gauthier et al., 2012). This method is finding growing adoption in the research setting, and has already been applied in Alzheimer's disease (Lajoie et al., 2017), carotid artery occlusion (De Vis et al., 2015), and studies of pharmacological modulation (Merola et al., 2017). For a review of the method and details on the its practical application please see Germuska and Wise (2019). Despite the promise shown by this technique, the reported between-session repeatability is relatively low (Merola et al., 2018) and improvements in the data acquisition and/or analysis are required if individualized assessment is to be made possible.

One of the key difficulties in analyzing dual-calibrated fMRI data is noise propagation through the analysis pipeline, which leads to unstable parameter estimates. We have previously presented regularized non-linear least squares fitting approaches that utilize prior physiological knowledge to produce more robust parameter estimates (Germuska et al., 2016, 2019). Even though such regularization reduces the mean square error it does so by trading off a reduction in variance with an increase in bias. An alternative approach to reduce the prediction error is the use of noise insensitive machine learning regression methods. Decision tree based regression methods, for example random forest (Breiman, 2001) and extremely randomized trees (Geurts et al., 2006), are robust to both output (Breiman, 2001; Geurts et al., 2006) and input noise (Yue et al., 2018) and are able to capture non-linear relationships between input features and target parameters. This noise immunity is likely due to the randomization included in the choices of features at splitting nodes (random forest) and cut-points (extremely randomized trees), which improve the generalizability of the regressors. For non-linear mappings with a high degree of complexity artificial neural networks, such as the multi-layer perceptron (MLP), a feedforward network with multiple hidden layers, offer a machine learning method that is inherently robust to noise (Bernier et al., 1999). In this paper we present an analysis pipeline comprised of an extremely randomized trees regressor and a MLP, cascaded to infer resting $\mathrm{CBF}$ and $\mathrm{CMRO}_{2}$ from dual-calibrated $\mathrm{fMRI}$ data. A frequency-domain representation of simulated MRI data with the additive noise is used to train each of the regressors. Simulated data has the advantage over in-vivo data in this application as it allows a balanced dataset to be generated that covers a broad range of physiological variation. Such a dataset is essential to avoid bias in parameter estimation and to provide generalizability across groups and diseases. A frequency-domain representation is chosen as it allows for convenient dimensionality reduction, with most of the information of interest encoded at low temporal frequencies, and takes advantage of the superior ability of artificial neural networks to learn discriminative features from frequency-domain representation of a signal compared to a timedomain representation (Hertel et al., 2016). The performance of the proposed machine learning (ML) implementation is compared to an existing regularized non-linear least squares (rNLS) method (Germuska et al., 2019) both in simulation and in data acquired from a cohort of 30 healthy volunteers. We hypothesized that the machine learning approach would be able to achieve comparable or reduced prediction error with significantly reduced bias and computational overhead.

\section{MRI DATA ACQUISITION}

Thirty healthy volunteers (16 males, mean age $32.53 \pm 6.06$ years) were recruited to the study, see Supplementary Table 1 for demographic data. The local ethics committee approved the study and written informed consent was obtained from each participant. Blood samples were drawn via a finger prick prior to scanning and were analyzed with the HemoCue $\mathrm{Hb}$ 301 System (HemoCue, Ängelholm, Sweden) to calculate the systemic $[\mathrm{Hb}]$ value for each participant. All data was acquired using a Siemens MAGNETOM Prisma (Siemens Healthcare $\mathrm{GmbH}$, Erlangen) 3T clinical scanner with a 32-channel receiver head coil (Siemens Healthcare GmbH, Erlangen). The acquisition protocol was as previously described (Germuska et al., 2019). Briefly, an 18-min dual-excitation pseudo-continuous arterial 
TABLE 1 | Range of physiological parameters used in the dc-fMRI data simulations for training of the machine learning regressors.

\begin{tabular}{|c|c|c|c|c|c|c|}
\hline OEF & $\begin{array}{l}\text { CBF } \\
\text { (ml/100g/min) }\end{array}$ & $\begin{array}{l}{[\mathrm{Hb}]} \\
\text { (g/dL) }\end{array}$ & $\begin{array}{l}\text { Mean capillary transit time } \\
\left(\mathrm{CBV}_{\text {cap }} / \mathrm{CBF}, \mathrm{s}\right)\end{array}$ & $\begin{array}{l}\mathrm{PminO}_{2} \\
(\mathrm{mmHg})\end{array}$ & $\begin{array}{l}\text { Cerebral vascular reactivity } \\
\left(\% \mathrm{CBF} / \mathrm{mmHg} \mathrm{CO}_{2}\right)\end{array}$ & $\mathbf{K}$ \\
\hline $0.05-0.75$ & $1-250$ & $10-18$ & $0.25-4.0$ & $0-30$ & $1-7$ & $0.01-0.25$ \\
\hline
\end{tabular}

spin labeling (pCASL) and BOLD-weighted acquisition was acquired during modulation of inspired oxygen and carbon dioxide. Gas modulation was performed according to a protocol previously proposed by our lab (Germuska et al., 2016), and end-tidal monitoring was performed throughout the acquisition from the volunteer's facemask using a rapidly responding gas analyzer (PowerLab $^{\circledR}$, ADInstruments, Sydney, Australia). The prototype pCASL sequence (Germuska et al., 2019) parameters were as follows: post-labeling delay and label duration $1.5 \mathrm{~s}$, EPI readout with GRAPPA acceleration (factor $=3$ ), $\mathrm{TE}_{1}=10 \mathrm{~ms}$, $\mathrm{TE}_{2}=30 \mathrm{~ms}, \mathrm{TR}=4.4 \mathrm{~s}, 3.4 \times 3.4 \mathrm{~mm}$ in-plane resolution, and $15(7 \mathrm{~mm})$ slices with $20 \%$ slice gap.

\section{SYNTHETIC MRI DATA GENERATION}

Synthetic data was simulated to match the 18-min in-vivo acquisition protocol using standard physiological models for the change in BOLD signal (Bulte et al., 2012; Gauthier and Hoge, 2013; Wise et al., 2013), as summarized by Equation (1).

$$
\frac{\triangle B O L D}{B O L D_{0}}=M\left\{1-\left(\frac{C B F}{C B F_{0}}\right)^{\alpha}\left(\frac{1-\frac{\mathrm{CaO}_{2}-C M R O_{2} / C B F}{\varphi[H b]}}{1-\frac{\mathrm{CaO}_{2,0}-C M R O_{2,0} / C B F_{0}}{\varphi[H b]}}\right)^{\beta}\right\}
$$

Where, $B O L D / B O L D_{0}$ is the fractional change in BOLD signal due to a change in arterial oxygen content $\left(\mathrm{CaO}_{2}\right)$ or $\mathrm{CBF}$ due to either a hyperoxic or hypercapnic respiratory stimulus. $\mathrm{M}$ is a lumped parameter that is equal to $K \cdot T E \cdot\left(\left(1-\mathrm{SvO}_{2}\right) \cdot[\mathrm{Hb}]\right)^{\beta}$. Where $\mathrm{K}$ is a scaling factor dependent on the field strength, resting venous blood volume, tissue structure, and water diffusion effects in the extravascular space. [Hb] is the blood hemoglobin concentration and $\mathrm{SvO}_{2}$ is the venous oxygen saturation. $\phi$ is the oxygen binding capacity for $\mathrm{Hb}(1.34 \mathrm{ml} / \mathrm{g})$, $\alpha$ is the Grubb exponent that couples blood volume and blood flow changes, and $\beta$ is a field strength dependent constant that summarizes the non-linear effects associated with the tissue structure and water diffusion effects. The values of $\alpha$ and $\beta$ were fixed to the optimized values (0.06 and 1) found by Merola et al. (2016), which minimize the error in OEF estimates over a range of vascular physiology. The subscript 0 represents the baseline or resting state. The hyperoxic and hypercapnic stimuli are assumed to be iso-metabolic, so $\mathrm{CMRO}_{2}=\mathrm{CMRO}_{2,0}$.

The arterial spin labeling signal was modeled according to the simplified pCASL kinetic model (Alsop et al., 2015), and physiological constraints on baseline parameters were applied according to a simple model of oxygen exchange (Gjedde, 2002;
Hayashi et al., 2003) (Equation 2).

$$
\mathrm{CMRO}_{2,0}=\mathrm{D}\left[\mathrm{P}_{50} \sqrt[h]{\frac{2}{\mathrm{OEF_{0 }}}-1}-P_{\min \mathrm{O}_{2}}\right]
$$

Where $\mathrm{D}$ is the effective oxygen diffusivity of the capillary network and can be expressed as a product of the effective oxygen permeability and the capillary blood volume, $D=\kappa \cdot C B V_{\text {cap }} . \mathrm{P}_{50}$ is the blood oxygen tension at which hemoglobin is $50 \%$ saturated (26 mmHg), $\mathrm{h}$ is the Hill coefficient (2.8) and $P_{\min \mathrm{O}_{2}}$ is the minimum oxygen tension at the mitochondria [which is thought to be negligible in healthy tissue (Gjedde, 2002)]. In the modeling we assume a fixed value for $\kappa$ of $3 \mu \mathrm{mol} / \mathrm{mmHg} / \mathrm{ml} / \mathrm{min}$, corresponding to a typical diffusivity of 3 (Mintun et al., 2001) to $4 \mu \mathrm{mol} / 100 \mathrm{~g} / \mathrm{mmHg} / \mathrm{min}$ (Vafaee and Gjedde, 2000) for $\mathrm{CBV}_{\text {cap }}=1-1.33 \mathrm{ml} / 100 \mathrm{~g}$. The physiological parameter space encompasses a wide range of plausible physiology including both healthy and dysfunctional brain tissue, and is summarized in Table 1. A summary of MRI abbreviations and all model parameters used in the simulations is given in Table 2 .

The partial pressure of arterial oxygen $\left(\mathrm{PaO}_{2}\right)$ and change in carbon dioxide $\left(\triangle \mathrm{PaCO}_{2}\right)$ were modeled to match the range of end-tidal recordings acquired from healthy volunteers. The baseline $\mathrm{PaO}_{2}$ had a range of $90-120 \mathrm{mmHg}, \Delta \mathrm{PaO}_{2}$ was 200 $300 \mathrm{mmHg}$, and $\triangle \mathrm{PaCO}_{2}$ was set to $8-12 \mathrm{mmHg}$. Rectangular stimulus blocks were convolved with a gamma density function with shape parameter $0.5-2.5$ to account for the variation in biological rise and fall times of the hyperoxic and hypercapnic stimuli. Drift in $\Delta \mathrm{PaCO}_{2}$, which was observed in some subjects, was included by adding a bandpass filtered noise signal (fourth order IIR filter, lowcut/highcut $=0.005 / 0.05$ of the Nyquist frequency). Change in the arterial blood longitudinal relaxation rate due to dissolved oxygen was included in pCASL calculations as per (Germuska et al., 2019). Noise (BOLD tSNR $=90$, pCASL $\mathrm{tSNR}=3$ for $\mathrm{CBF}=60 \mathrm{ml} / 100 \mathrm{~g} / \mathrm{min}$ ) was added to simulated BOLD and pCASL time series. The pCASL noise was bandpass filtered (fourth order IIR filter, lowcut/highcut $=0.05 / 0.8$ of the Nyquist frequency) and the BOLD noise was lowpass filtered (first order IIR filter, highcut $=0.5$ of the Nyquist frequency) to match the noise characteristics of the in-vivo data. In addition, the BOLD timeseries data was highpass filtered with a $320 \mathrm{~s}$ cutoff using the filter implementation in FSL (Jenkinson et al., 2012), which is routinely used for de-trending fMRI data. Figure 1 shows 50 randomly generated pCASL and BOLD timeseries overlaid with the temporal mean to demonstrate the typical output of the simulations. Please note that the pCASL timeseries are divided by the equilibrium magnetization of arterial blood $\left(\mathrm{M} 0_{\text {blood }}\right)$, and the baseline signal has been set to zero for display purposes. 
TABLE 2 | Summary of model parameters and abbreviation used in the dc-fMR| data simulations and their definitions.

\begin{tabular}{|c|c|}
\hline $\begin{array}{l}\text { Variable/ } \\
\text { abbreviation }\end{array}$ & Expression (units) \\
\hline OEF & Oxygen Extraction Fraction (dimensionless) \\
\hline $\mathrm{CMRO}_{2}$ & Cerebral Metabolic Rate of Oxygen consumption ( $\mu \mathrm{mol} / 100 \mathrm{~g} / \mathrm{min})$ \\
\hline CBF & Cerebral Blood Flow (ml/100 g/min) \\
\hline$\phi$ & Oxygen binding capacity of hemoglobin $(1.34 \mathrm{ml} / \mathrm{g})$ \\
\hline$[\mathrm{Hb}]$ & Hemoglobin concentration (g/dL) \\
\hline $\mathrm{CaO}_{2}$ & Arterial oxygen content (ml/ml) \\
\hline $\mathrm{PaO}_{2}$ & Arterial oxygen tension $(\mathrm{mmHg})$ \\
\hline $\mathrm{SaO}_{2}$ & Arterial oxygen saturation (dimensionless) \\
\hline $\mathrm{SvO}_{2}$ & Venous oxygen saturation (dimensionless) \\
\hline$\alpha$ & Grubb exponent \\
\hline$\beta$ & Venous morphology/deoxy-hemoglobin-BOLD exponent \\
\hline BOLD & Blood Oxygenation Level Dependent signal \\
\hline ASL & Arterial Spin Labeling \\
\hline $\mathrm{MO}_{\text {blood }}$ & Arterial blood MRI signal equilibrium magnetization (dimensionless) \\
\hline PLD & ASL post-label delay time (1.0-3.0 s) \\
\hline M & Maximum possible BOLD signal (BOLD calibration parameter) \\
\hline K & BOLD scaling factor $=\mathrm{M} /\left([\mathrm{Hb}] \times\left(1-\mathrm{SvO}_{2}\right)\right)^{\beta}$ \\
\hline $\mathrm{D}$ & $\begin{array}{l}\text { Effective oxygen diffusivity of the capillary network ( } \mu \mathrm{mol} / 100 \\
\mathrm{~g} / \mathrm{mmHg} / \mathrm{min} \text { ) }\end{array}$ \\
\hline $\mathrm{CBV}_{\text {cap }}$ & Capillary blood volume (ml/100 g) \\
\hline $\mathrm{PminO}_{2}$ & Minimum oxygen partial pressure at the mitochondria $(\mathrm{mmHg})$ \\
\hline $\mathrm{h}$ & Hill coefficient (2.8) \\
\hline$\kappa$ & $\begin{array}{l}\text { Effective permeability of capillary endothelium and brain tissue } \\
(\mu \mathrm{mol} / \mathrm{mmHg} / \mathrm{ml} / \mathrm{min})\end{array}$ \\
\hline
\end{tabular}

\section{METHODS}

A schematic diagram describing the analysis/training pipeline is shown in Figure 2. ASL and BOLD timeseries data, either simulated (as described in section Synthetic MRI Data Generation) or in-vivo data, are Fourier transformed into magnitude and phase data. This frequency domain data is then truncated after the first 15 data points (low pass filtered) and combined with physiological recordings and sequence parameters to create a feature vector for model training/prediction (if in-vivo data is being analyzed). Parameter estimation is carried out in a two-stage process; first the resting blood flow $\left(\mathrm{CBF}_{0}\right)$ is estimated, and then rate of oxygen consumption.

Truncation of the frequency domain data removes highfrequency content that is unrelated to either the hyperoxic or hypercapnic respiratory modulations and thus removes noise from the training data. The resting blood flow is estimated separately from the rate of oxygen consumption to reduce the complexity of the required mapping between the MRI data and the target parameters. Additionally, the use of extremely randomized trees (ET) regression rather than an artificial neural network at this stage in the pipeline takes full advantage of the noise immunity of decision tree based methods (Yue et al., 2018) and reduces the potential of overfitting. The inclusion of the
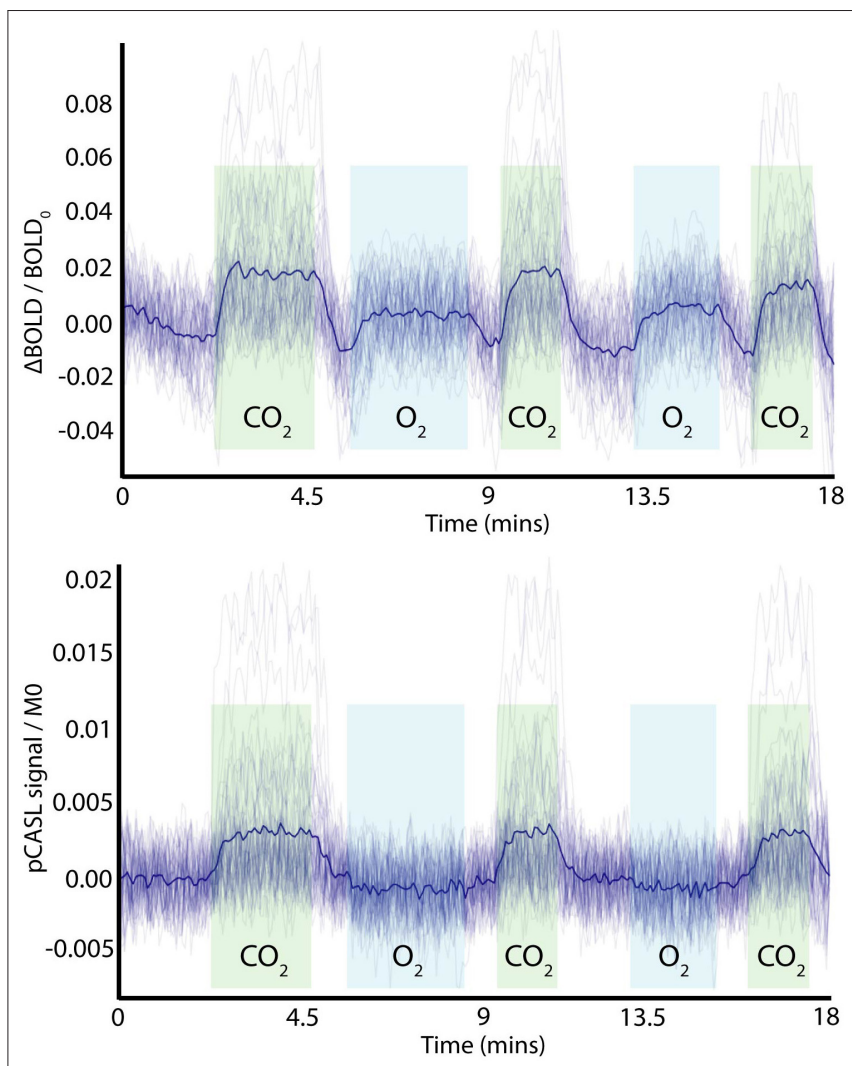

FIGURE 1 | Example of simulated time-domain data (BOLD and ASL) with added noise and variation in physiological parameters, showing periods of hypercapnic (green) and hyperoxic (light blue) stimuli. The dark blue line represents the mean time-course over the example time series. Note the pCASL signal is normalized by the equilibrium magnetization of arterial blood (MO) and has had the baseline signal subtracted for display purposes.

post-label delay in the feature vector is necessary to incorporate an implicit slice timing correction for $\mathrm{CBF}_{0}$ calculation, while the blood oxygenation parameters ( $\left.[\mathrm{Hb}], \Delta \mathrm{PaO}_{2}, \mathrm{SaO}_{2,0}, \mathrm{CaO}_{2,0}\right)$ are included here due to the influence of dissolved oxygen on the longitudinal relaxation rate of arterial blood. In total each feature vector that is input into the ET regressor consists of 65 entries.

The result of the ET regression is then incorporated into the feature vector (now 66 entries) and input into an ensemble of MLPs to predict $\mathrm{CMRO}_{2,0} / \mathrm{CaO}_{2,0}$, from which $\mathrm{CMRO}_{2,0}$ and $\mathrm{OEF}_{0}$ can be calculated $\left(\mathrm{CMRO}_{2} / \mathrm{CaO}_{2}=\mathrm{OEF} \times \mathrm{CBF}\right.$ via the Fick principle). The blood oxygenation parameters in this case not only inform on the relaxation rate of arterial blood, but also link the CBF and BOLD signal changes to the underlying metabolic parameters as described by Equation (1). In practice each MLP in the ensemble is trained individually, with the average of their predictions being used for inference when deployed for the analysis of in-vivo data.

The ET regressor and MLP were implemented in Scikit learn (Pedregosa et al., 2011). The extremely randomized trees regressor was trained with the following options, number of estimators $=50$, bootstrap $=$ True, and out-of-bag samples were used to estimate the $R^{2}$ on unseen data. A total of 50,000 


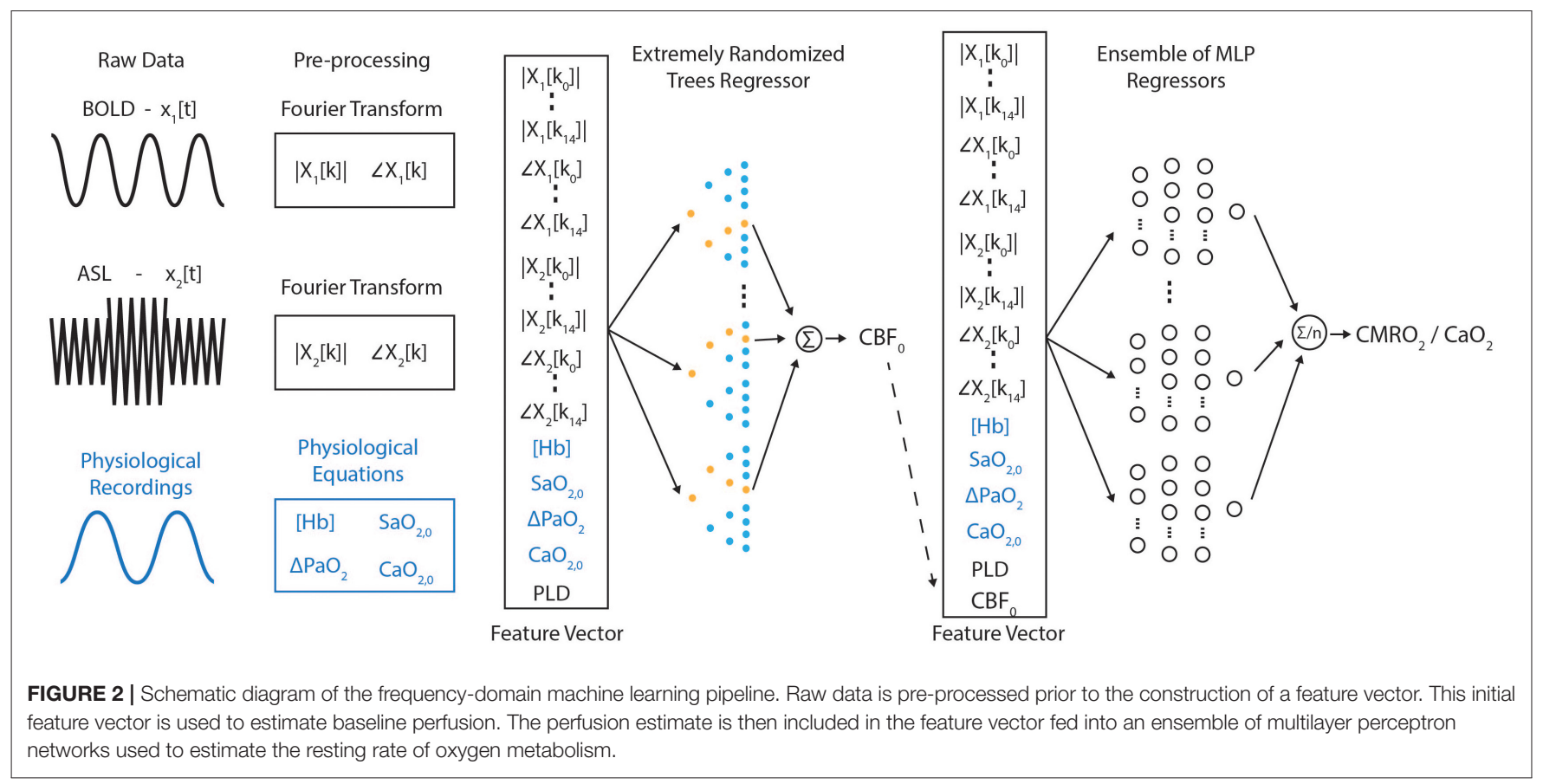

simulations were used for training. The MLP network has twohidden layers and 50 nodes in each layer. The activation function for each node was chosen to be a rectified linear unit (ReLU). The ADAM solver was used for training with $1 \times 10^{6}$ simulated feature vectors and $10 \%$ of the data were used for early stopping. Data simulation and training was repeated 40 times to create an ensemble of MLP networks to further reduce the uncertainty in parameter estimates (Sollich and Krogh, 1996).

The validation score for the extremely randomized trees regressor for predicting resting cerebral blood flow was 0.997 , slightly greater than the results obtained for a random forest implementation (0.961). The validation score for the MLP estimation of $\mathrm{CMRO}_{2,0} / \mathrm{CaO}_{2,0}$ were $0.923 \pm 0.002$. Training of the MLP network was also undertaken while eliminating key elements of the simulation or feature vectors to see how this affected the performance of the MLP. When BOLD data was excluded from the feature vector the validation score dropped to 0.577. Excluding the $\mathrm{CO}_{2}$ and $\mathrm{O}_{2}$ stimuli (but including the BOLD data) reduced the validation scores to 0.63 and 0.71 , respectively.

A further 5,000 simulated datasets (with OEF restricted to 0.15-0.65, all other parameters as in Table 1) were constructed to compare the performance of the proposed machine learning implementation with a previously implemented regularized nonlinear least squares fitting method (Germuska et al., 2019). Each method was compared to the simulated data using a robust regression method (bisquare) in terms of the RMS error and proportional bias. A bisquare cost function was used for the regression to reduce the influence of outliers and allow a robust estimate of the proportional bias. The rNLS fitting was implemented with regularization applied to the resting OEF and the effective oxygen diffusivity (D), as previously described. The relative weighting between $\mathrm{OEF}$ and diffusivity regularization was maintained constant, as per the optimization in Germuska et al. (2019). However, the total weighting was varied to assess the impact on OEF and $\mathrm{CMRO}_{2}$ error and proportional bias (slope of the simulated parameter values plotted against the parameter estimates).

\section{RESULTS}

\section{Simulations}

Analysis of the simulated data demonstrated a substantial reduction in the RMS error of machine learning OEF estimates compared to rNLS estimates. The bisquare RMS error was 0.047 when using the mean prediction from the 40 MLP networks, and 0.055 for a randomly chosen MLP network. The rNLS approach produced a minimum bisquare RMS error of 0.094 . The ML approach displayed negligible proportional bias in OEF estimates (slope of true vs. estimated values $=0.982$ ), whereas rNLS estimates had variable levels of bias depending on the level of regularization, see Figure $\mathbf{3 A}$ for a summary of the results. As expected from the OEF results, ML estimates of $\mathrm{CMRO}_{2}$ also had significantly reduced error and bias compared to the rNLS implementation. The proportional bias for the ML implementation was 0.977 compared to a minimum bias of 0.913 for the rNLS method. The bisquare RMS error in $\mathrm{CMRO}_{2}$ estimates for the ML implementation was $20.3 \mu \mathrm{mol} / 100 \mathrm{~g} / \mathrm{min}$ (22.6 for an individual MLP network) whereas the error for rNLS estimates ranged from 29.6 to $52.4 \mu \mathrm{mol} / 100 \mathrm{~g} / \mathrm{min}$ depending on the level of bias (with greater bias coinciding with reduced error) (see Figure 3B).

Training of the MLP with reduced feature vectors (excluding the BOLD data) or limited respiratory stimuli (excluding either 

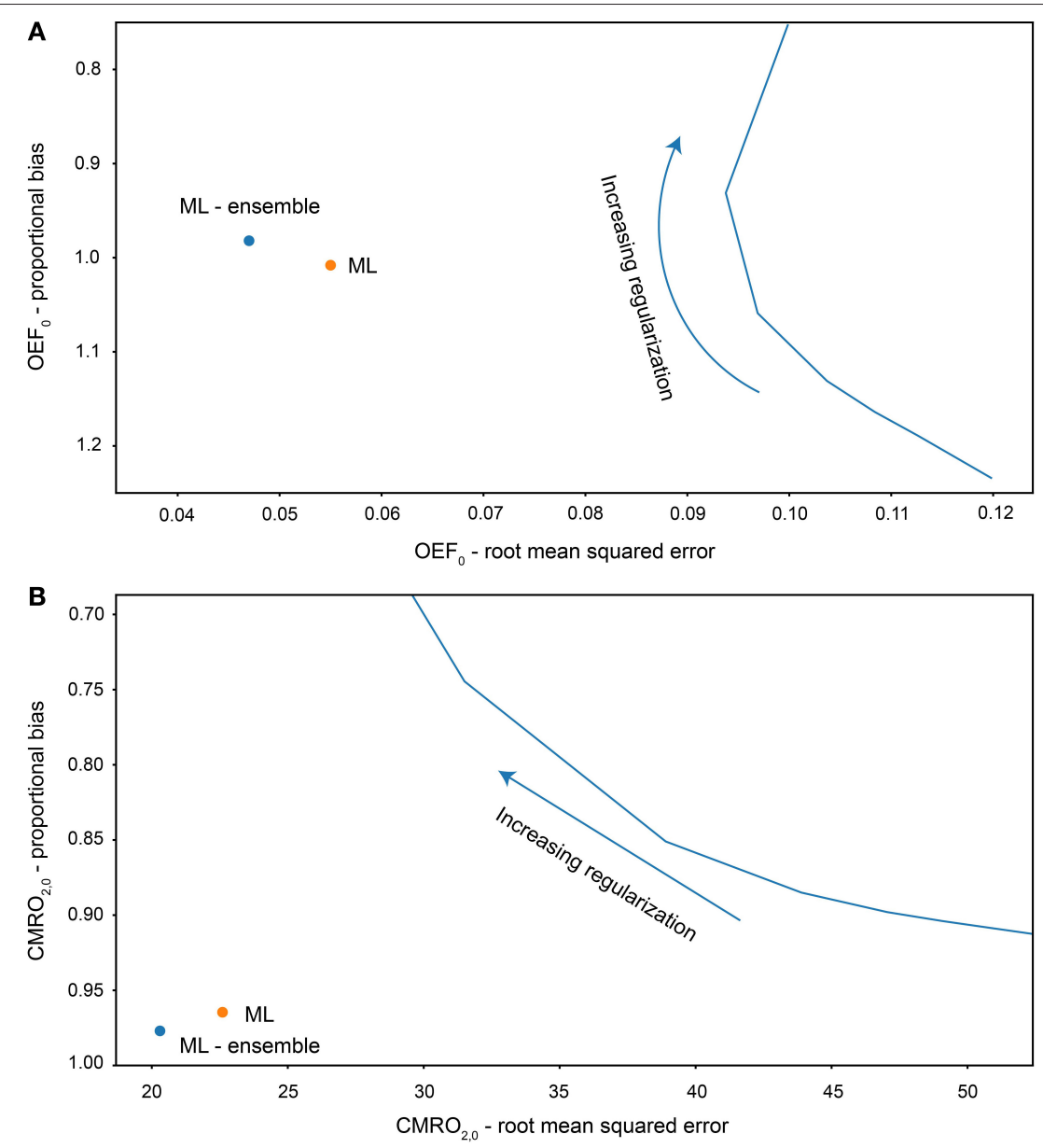

FIGURE 3 | Root mean squared error and proportional bias in $\mathrm{OEF}_{0}$ (A) and $\mathrm{CMRO}_{2,0}$ (B) estimates for each analysis method fitting to simulated data (5,000 simulations). Solid blue line plots the error and bias for increasing regularization weighting for the regularized non-linear least squares analysis.

$\mathrm{CO}_{2}$ or $\mathrm{O}_{2}$ modulation) highlights the importance of each signal and stimulus in estimate the rate of oxygen consumption. As expected, removing the BOLD signal resulted in a significant reduction in the network's ability to estimate $\mathrm{CMRO}_{2}$ (validation $R^{2}$ reduced from 0.923 for the full model to 0.58 ). In this instance there should be no information relating to OEF in the feature vector and so the inference is based solely on the correlation between baseline flow and $\mathrm{CMRO}_{2}$ in the simulated data. Adding the BOLD data back in but with only an $\mathrm{O}_{2}$ stimulus does little to improve the performance of the network $\left(R^{2}=0.63\right)$. This is not unexpected as the hyperoxic BOLD signal is largely related to venous blood volume (Blockley et al., 2013) with little influence from OEF. Perhaps unexpectedly, including the $\mathrm{CO}_{2}$ stimulus but not the $\mathrm{O}_{2}$ stimulus significantly improves the ability of the network to infer resting $\mathrm{CMRO}_{2}\left(R^{2}=0.71\right)$. While this is still significantly worse than the full model, it suggests that some quantitative metabolic information may be extracted from hypercapnic calibration studies that are normally employed to estimate relative changes in $\mathrm{CMRO}_{2}$ (Hoge, 2012). Additionally, such results suggest that the simulation framework could be utilized to optimize data acquisition by designing respiratory stimuli that maximize the performance of the ML implementation, and that such respiratory paradigms may be different compared to those for standard analysis methods (which are unable to infer resting $\mathrm{CMRO}_{2}$ information from a hypercapnic calibration experiment).

\section{In-vivo}

Due to the limited availability and technical challenges associated with acquiring 15-oxygen PET data for $\mathrm{CMRO}_{2}$ mapping (the gold standard approach) it is difficult to directly validate the in-vivo results obtained in this study. However, a number of fundamental relationships between resting physiological parameters have consistently been observed across groups of healthy individuals. Here we compare these observed relationships against the acquired data to infer the relative error and bias for each analysis method. One of the most frequently reported relationships in the healthy human brain is that resting blood flow is linearly correlated with resting oxygen metabolism (Scheinberg and Stead, 1949; Lebrun-Grandie et al., 1983; Leenders et al., 1990; Coles et al., 2006; Powers et al., 2011). Additionally, PET data suggests that the OEF should 

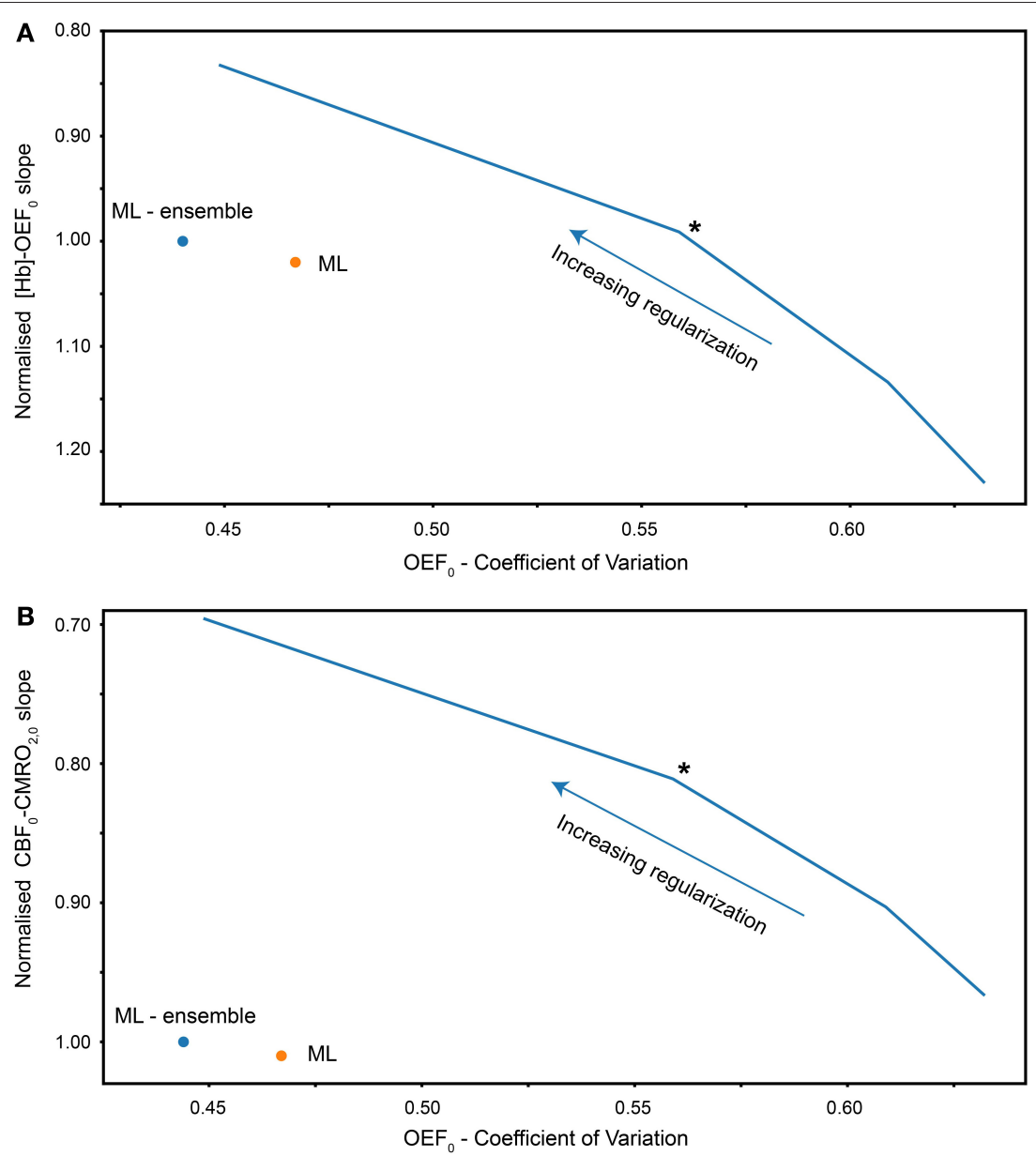

FIGURE 4 | (A) Coefficient of variation of gray matter OEFo estimates vs. slope of [Hb]-OEFo relationship for each analysis method (rNLS fitting evaluated with increasing levies of regularization). The $[\mathrm{Hb}]-\mathrm{OEF}_{0}$ slope has been normalized by the $\mathrm{ML}$ ensemble estimate of the [Hb]-OEF $\mathrm{H}_{0}$ slope. (B) Coefficient of variation of gray matter $\mathrm{OEF}_{0}$ estimates vs. the slope of the $\mathrm{CBF}-\mathrm{CMRO}_{2}$ relationship, normalized by the $\mathrm{ML}$ (ensemble) estimate of the $\mathrm{CBF}^{-\mathrm{CMRO}} 2$ slope. Solid blue line plots the coefficient of variation against the slope for increasing regularization weighting for regularized non-linear least squares analysis. The asterisk indicates the chosen level of regularization for subsequent analysis/comparisons.

be approximately uniform across the cerebral gray matter (e.g., Hyder et al., 2016). Thus, we can use the coefficient of variation $(\mathrm{COV})$ of gray matter OEF estimates as an indicator of parameter error, and examine the variation in the slope of the CBF$\mathrm{CMRO}_{2}$ relationship to infer the proportional bias or sensitivity to physiological variation of $\mathrm{CMRO}_{2}$ estimates.

As in the simulation experiments we investigated the in-vivo analysis for varying levels of regularization in the rNLS analysis and compare this to the ML results. Figure $4 \mathrm{~B}$ plots the COV in $\mathrm{OEF}$ estimates for increasing levels of regularization against the slope of the $\mathrm{CBF}-\mathrm{CMRO}_{2}$ regression (normalized by the slope of the ML estimate). As predicted by the simulations, the slopes of the ML estimates and the rNLS estimates are similar when little regularization is applied, with the slope of the rNLS estimates slightly reduced compared to the ML approach. As more regularization is applied the $\mathrm{COV}$ of OEF estimates is reduced and the slope between $\mathrm{CBF}$ and $\mathrm{CMRO}_{2}$ decreases, clearly demonstrating the trade-off between variance and bias.
Again, as predicted by the simulations, the COV in ML estimates is significantly less than COV in rNLS estimates for a similar $\mathrm{CBF}-\mathrm{CMRO}_{2}$ slope.

To investigate the bias in OEF estimates we take advantage of another physiological relationship reported in the literature; cerebral oxygen extraction is inversely related to $[\mathrm{Hb}$ ] (Ibaraki et al., 2010) and the closely related parameter Hct (Morris et al., 2018). Taking the same approach as before we observe invivo results that closely match predictions from the simulation (see Figure 4A). As in the simulations, the slope in the $[\mathrm{Hb}]-\mathrm{OEF}$ relationship is similar between the ML method and rNLS approach for a moderate amount of regularization. However, the slope is substantially increased when using minimal regularization, and reduced when applying strong regularization.

Figure 5 shows scatter plots of the gray matter $\mathrm{CBF}-\mathrm{CMRO}_{2}$ and $[\mathrm{Hb}]-\mathrm{OEF}$ relationships observed with the ML and rNLS methods across the 30 healthy volunteers studied. The rNLS results are shown for a single level of regularization, where the 

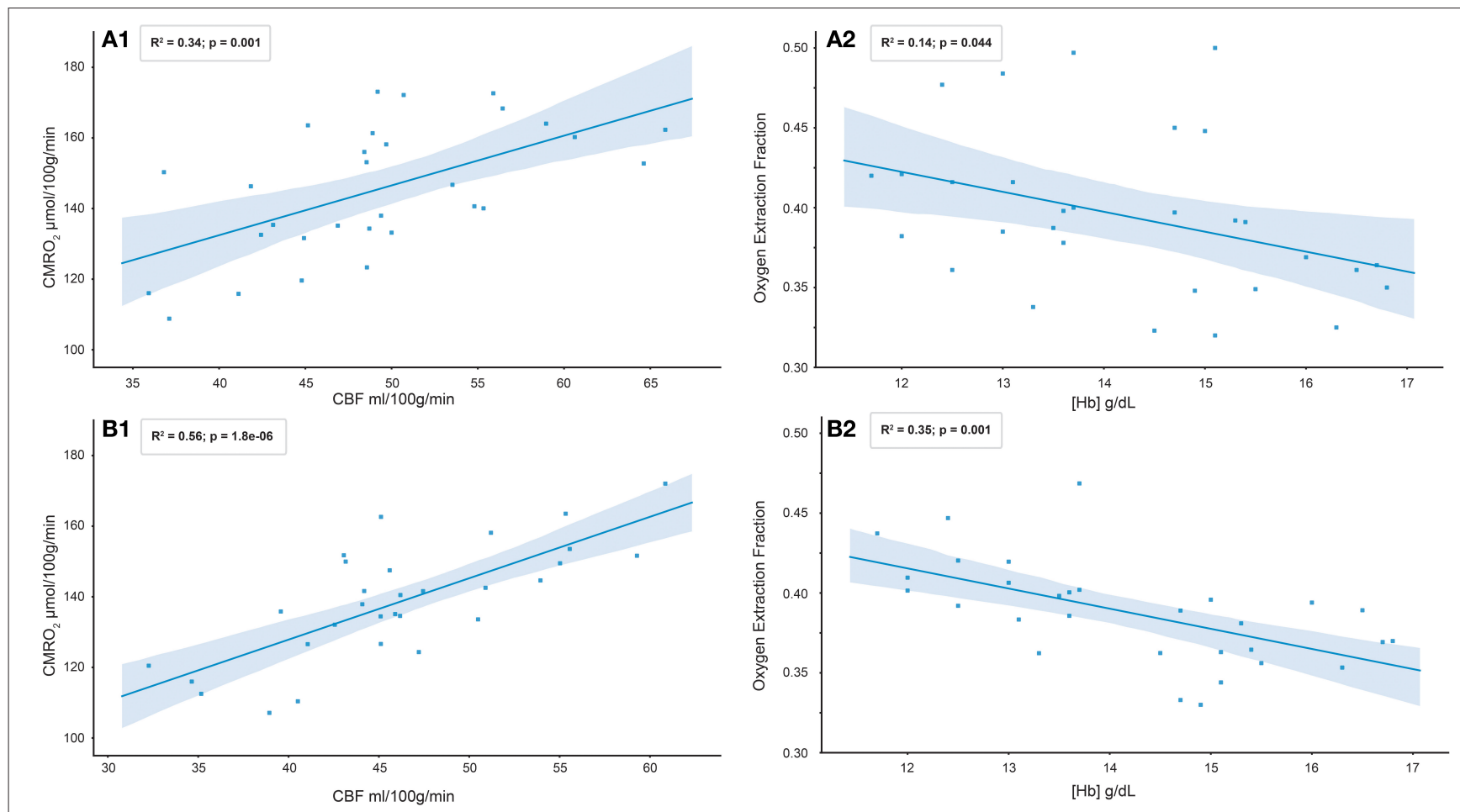

FIGURE 5 | Scatter plots of gray matter $\mathrm{C} 8 \mathrm{~F}-\mathrm{CMRO}_{2}$ and [Hb]-OEF relationships observed with $\mathrm{rNLS}$ (A1 and $\left.\mathrm{A} 2\right)$ and ML ensemble (B1 and B2) methods across 30 healthy volunteers.

slope of the $[\mathrm{Hb}]-\mathrm{OEF}$ relationship most closely matches that of the ML analysis (see Figure 4). The coefficient of determination is greater for the ML approach for each relationship, with $R^{2}$ values of 0.56 and 0.35 for the $\mathrm{CBF}-\mathrm{CMRO}_{2}$ and [Hb]-OEF relationships, compared to 0.34 and 0.14 for the rNLS approach ( $p<0.05$ for all correlations).

Table 3 reports the results of a bivariate regression of OEF against $[\mathrm{Hb}]$ and $\mathrm{CBF}$ for both analysis methods. The slopes of the relationship between $\mathrm{OEF}$ and $[\mathrm{Hb}]$ are similar to that reported in healthy subjects by (Ibaraki et al., 2010), $-1.75 \mathrm{Hb}$ $(\mathrm{g} / \mathrm{dL})$. As per Ibaraki et al. the relationship between $\mathrm{CBF}$ and OEF did not reach significance $(p=0.44)$ for the ML approach, however a significant negative correlation was observed in the rNLS analysis $(p=0.005)$. A univariate analysis of $\mathrm{CMRO}_{2,0}$ against $\mathrm{CBF}_{0}$ is consistent with that observed in healthy controls by (Powers et al., 2011) $(\beta 1=0.2)$ for both analysis methods, $\beta 1$ $=0.32(p<0.001)$ and $\beta 1=0.24(p<0.001)$ for the ML and rNLS approaches respectively.

Figure 6 shows a comparison between $\mathrm{CBF}_{0}, \mathrm{OEF}_{0}$, and $\mathrm{CMRO}_{2,0}$ parameter maps calculated with the ML method (single MLP network and ensemble of 40 networks) and the rNLS method. The image shows seven slices from a single subject, which have been interpolated for display using cubic b-spline interpolation (Ruijters and Thevenaz, 2012) using FSLeyes (10.5281/zenodo.1470761). As expected $\mathrm{OEF}_{0}$ is not well-estimated in the white matter, due to the $\mathrm{T}_{1}$ decay of the arterial spin labeling signal and the longer arrival time of white matter blood. Across gray matter containing voxels maps of $\mathrm{OEF}_{0}$
TABLE 3 | Results of a bivariate regression of $\mathrm{OEF}_{0}$ against $\mathrm{CBF}_{0}$ and $[\mathrm{Hb}]$ for 30 healthy volunteers analyzed with the ML (ensemble of MLPS) and rNLS fitting methods.

\begin{tabular}{lcc}
\hline Predictor & ML $\boldsymbol{\beta} \mathbf{1}$ ( $\boldsymbol{p}$ value) & rNLS $\boldsymbol{\beta} \mathbf{1}$ ( $\boldsymbol{p}$ value) \\
\hline$[\mathrm{Hb}]$ & $-1.42(0.001)$ & $-2.23(0.001)$ \\
$\mathrm{CBF}$ & $-0.07(0.44)$ & $-0.37(0.005)$ \\
Intercept & $61.95(<0.001)$ & $89.48(<0.001)$ \\
\hline
\end{tabular}

calculated with the ML methods are more uniform than those calculated with the rNLS approach, with the ensemble approach visibly outperforming the singe network MLP estimates. These observations are consistent with the results of the simulations and the gray matter COV observed for in-vivo $\mathrm{OEF}_{0}$ estimates. However, it is also apparent from the images that each method demonstrates sensitivity to regional susceptibility effects. For example, in the pre-frontal cortex and inferior temporal lobes the images show greater variability in $\mathrm{OEF}_{0}$ estimates, with regions of both over and under-estimation apparent. This instability is likely due to reduced BOLD SNR in these locations and alteration of the susceptibility of air in and around the nasal cavity and paranasal sinuses due to modulation of the inspired oxygen content during data acquisition. It is clear that the ML estimates, in particular those made from the ensemble of MLPs, are more robust to such regional susceptibility effects.

The in-vivo analysis also highlights the improvement in computational efficiency of the proposed method. The rNLS 

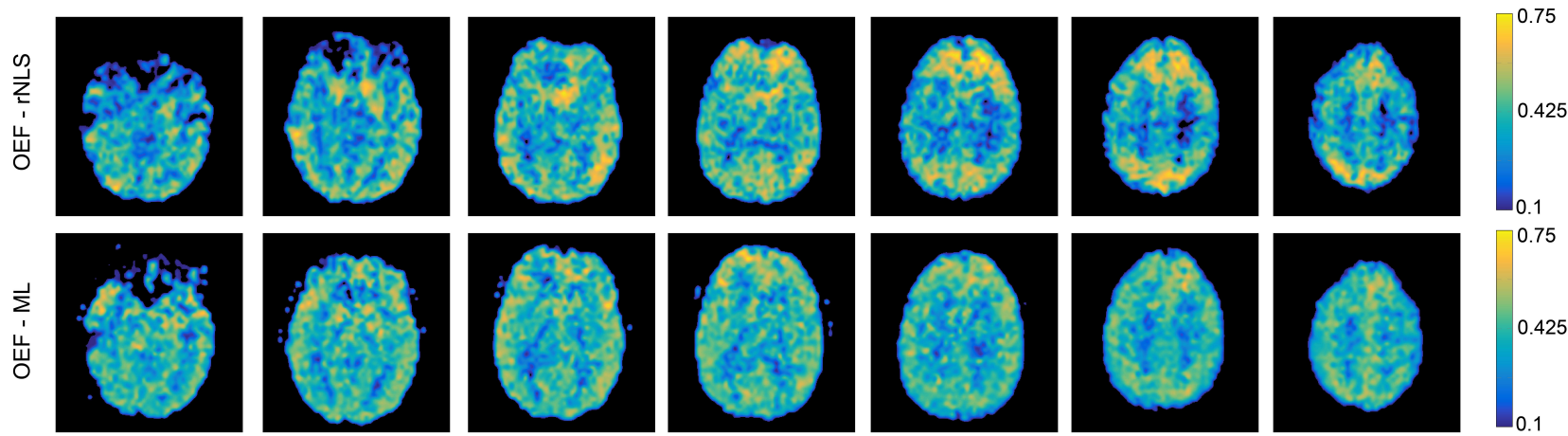

$\square 0.75$
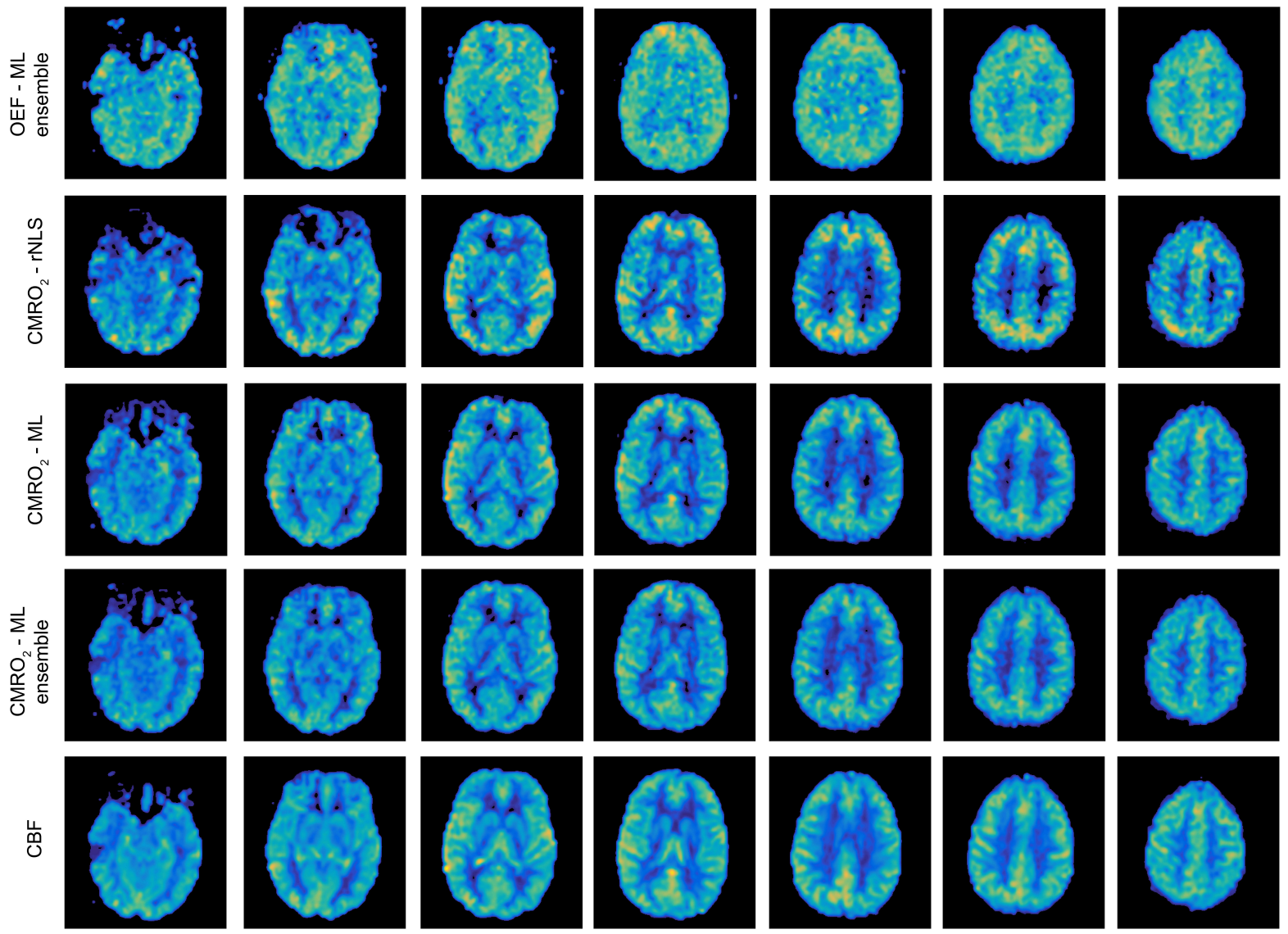

FIGURE 6 | Example parameter maps $\left(\mathrm{CBF}_{0}, \mathrm{OEF}_{0}\right.$, and $\left.\mathrm{CMRO}_{2,0}\right)$ from a single subject for each analysis method. Machine learning estimates of $\mathrm{OEF}$ o are more

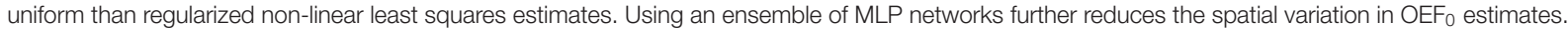

approach took $\sim 20$ min to analyze a complete dataset on a standard laptop (2.8 Ghz Intel Core i7, 16GB memory), while the ML approach was able to complete the same analysis in $\sim 10$ $20 \mathrm{~s}$ (depending on the number of networks in the ensemble of MLP regressors).

\section{DISCUSSION AND CONCLUSIONS}

Instability in parameter estimates made using noisy in-vivo data may be reduced by incorporating prior knowledge of physiological parameters (e.g., Chappell et al., 2010; Frau-Pascual et al., 2014; Mesejo et al., 2015; Germuska et al., 2016). Previous investigation of such methods (Germuska et al., 2016) suggests that they are an effective means to increase the robustness of $\mathrm{CMRO}_{2}$ estimates made with dc-fMRI. However, these methods are computationally expensive and must necessarily make a trade off between parameter uncertainty and parameter sensitivity. Thus, they are not well-suited to high throughput or rapid data analysis and care must be taken when using such methods not to unduly bias parameter estimates toward the priors. In the work presented here we take a different approach by training a machine learning implementation that is robust to input noise. 
Given an appropriately selected (or generated) training dataset, a well-implemented solution will be unbiased, robust, and have a low computational overhead.

Computer modeling suggests that the proposed method outperforms previous analysis methods both in terms of uncertainty and bias. In-vivo data supports the predicted improvement in uncertainty with a significant reduction in the COV of gray matter $\mathrm{OEF}_{0}$ estimates when compared to a regularized non-linear least squares fitting of the data. Additionally, agreement was found between the predicted behaviors of each method and their associated biases when compared to reported physiological relationships. Qualitatively, the in-vivo parameter maps suggest that the ML approach, especially when paired with an ensemble implementation, is more robust to physiological noise; producing physiologically plausible parameter estimates in challenging brain regions, e.g., near the frontal sinuses. Such physiological noise was not modeled in the training data so it is perhaps unexpected that the ML method is robust to these noise sources. However, it is plausible that the discriminative features identified from the frequency-domain representation of the data during training are less sensitive to these regional susceptibility changes than a traditional time-domain fit of the data. It is possible that this aspect of the ML approach could be enhanced by extending the training data to include such regional susceptibility changes, either on their own or in combination with a spatially informed approach to data fitting.

The use of an ensemble of MLP networks reduced parameter uncertainty in simulation and reduced the coefficient of variation in gray matter $\mathrm{OEF}_{0}$ estimates in-vivo, demonstrating its utility in this application. However, it is anticipated that enforcing network diversity during training could make further improvements in performance. As it is has previously been demonstrated that, in the presence of noise, the performance of an ensemble of networks can always be improved by explicitly encouraging diversity during training (Reeve and Brown, 2018).

The machine learning implementation presented here employs a combination of proven signal processing (timefrequency transformation) and machine learning methods (decision trees and fully connected artificial neural networks) that have been shown to select appropriate features for learning and are robust to input noise. The proposed analysis pipeline demonstrates an improvement in both the accuracy and precision in parameter estimates compared to published methods, and is appropriate for the study of both healthy volunteers and in clinical investigations. However, there are still many avenues that could be explored both in terms of signal processing and machine-learning. For example time domain data could be converted to 2D time-frequency representations, such as a spectrogram, or into spectrogram-like representations using wavelet transforms (for increased time resolution). This type of pre-processing would open the door to the application of $2 \mathrm{D}$ convolution neural networks $(\mathrm{CNN})$ that have been so successfully applied in the domain of image processing. It is possible that the application of such approaches could further improve the performance of machine learning when analyzing dc-fMRI data. However, a thorough investigation of all available machine learning methods and associated pre-conditioning of the data is beyond the scope of the current study, which focuses instead on the realization of a practical solution by combining well-proven techniques for the analysis of signal data.

All in-vivo analysis in this manuscript is performed in the absence of spatial smoothing, which is often employed to improve statistical estimates made from fMRI data (Friston et al., 1995). We chose not to employ spatial smoothing in this analysis for two principle reasons: first any such spatial filtering implies a prior assumption regarding the spatial extent of any variation (Rosenfeld and Kak, 1982), and can thus lead to unwanted loss of sensitivity to physiological variation; second we did not want to increase the potential contamination of gray matter voxels with non-tissue signals, such as CSF or macrovessels (both of which are not included in the underlying signal model). The current study does not make any direct comparison between smoothed and unsmoothed analysis pipelines, however the presented method clearly avoids any possible smoothing artifacts that might otherwise bias the analysis.

A limitation of the proposed method is the need to train new regressors for a given gas paradigm and set of acquisition parameters, e.g., arterial spin labeling tagging duration, repetition time and duration of the acquisition. In addition, there is a requirement that the in-vivo gas manipulation does not deviate significantly from the range of simulated designs. While it is a relatively straightforward process to retrain the regressors with a new set of parameters, to match the local acquisition protocol, the scope of the method could be increased if individualized gas traces could be incorporated into the training data; allowing a single pre-trained implementation to be applied across studies.

The simulations and in-vivo results suggest that the proposed analysis method could significantly increase the utility of dcfMRI, reducing the number of participants needed to detect a group difference in oxygen metabolism or oxygen extraction fraction and offering more physiological interpretability of metabolic differences or alteration due to a stimulus. In addition, the significant reduction in processing time and the improved robustness of the individual parameter maps reduces two of the hurdles restricting clinical implementation of such techniques.

\section{DATA AVAILABILITY STATEMENT}

The python code for the machine learning implementation proposed in this article can be found in the fml_pMRI repository https://zenodo.org/badge/latestdoi/189416118. We do not have ethical consent to make the in-vivo datasets acquired for this study publically available.

\section{ETHICS STATEMENT}

The studies involving human participants were reviewed and approved by School of Psychology Research Ethics Committee Cardiff University. The patients/participants provided their written informed consent to participate in this study. 


\section{AUTHOR CONTRIBUTIONS}

MG wrote the manuscript, developed and implemented the methods, and analyzed the in-vivo data. $\mathrm{HC}$ acquired and processed the data and edited the manuscript. TO created and provided the code used in the prototype pseudo-continuous arterial spin labeling pulse sequence. FF assisted in the implementation of the prototype arterial spin labeling pulse sequence. VT was the chief investigator overseeing the study design and data collection for a subset of healthy controls. $\mathrm{KM}$ and RW was the principal investigator overseeing the study design and data collection for a subset of healthy controls. All authors reviewed and edited the manuscript prior to submission.

\section{FUNDING}

This study was funded by Wellcome Strategic Award, Multiscale and multi-modal assessment of coupling in the healthy and diseased brain, grant reference 104943/Z/14/Z (RW,

\section{REFERENCES}

Alsop, D. C., Detre, J. A., Golay, X., Gunther, M., Hendrikse, J., HernandezGarcia, L., et al. (2015). Recommended implementation of arterial spin-labeled perfusion MRI for clinical applications: a consensus of the ISMRM perfusion study group and the European consortium for ASL in dementia. Magn. Reson. Med. 73, 102-116. doi: $10.1002 / \mathrm{mrm} .25197$

Bernier, J. L., Ortega, J., Ros, E., Rojas, I., and Prieto, A. (1999). A new measurement of noise immunity and generalization ability for MLPs. Int. J. Neural. Syst. 9, 511-521. doi: 10.1142/S0129065799000551

Blockley, N. P., Griffeth, V. E., Germuska, M. A., Bulte, D. P., and Buxton, R. B. (2013). An analysis of the use of hyperoxia for measuring venous cerebral blood volume: comparison of the existing method with a new analysis approach. Neuroimage 72, 33-40. doi: 10.1016/j.neuroimage.2013. 01.039

Breiman, L. (2001). Random forests. Mach. Learn. 45, 5-32. doi: 10.1023/A:1010933404324

Bulte, D. P., Kelly, M., Germuska, M., Xie, J., Chappell, M. A., Okell, T. W., et al. (2012). Quantitative measurement of cerebral physiology using respiratory-calibrated MRI. Neuroimage 60, 582-591. doi: 10.1016/j.neuroimage.2011.12.017

Chappell, M. A., MacIntosh, B. J., Donahue, M. J., Gunther, M., Jezzard, P., and Woolrich, M. W. (2010). Separation of macrovascular signal in multiinversion time arterial spin labelling MRI. Magn. Reson. Med. 63, 1357-1365. doi: $10.1002 / \mathrm{mrm} .22320$

Coles, J. P., Fryer, T. D., Bradley, P. G., Nortje, J., Smielewski, P., Rice, K., et al. (2006). Intersubject variability and reproducibility of 150 PET studies. J. Cereb. Blood Flow. Metab. 26, 48-57. doi: 10.1038/sj.jcbfm.9600179

De Vis, J. B., Petersen, E. T., Bhogal, A., Hartkamp, N. S., Klijn, C. J., Kappelle, L. J., et al. (2015). Calibrated MRI to evaluate cerebral hemodynamics in patients with an internal carotid artery occlusion. J. Cereb. Blood Flow. Metab. 35, 1015-1023. doi: 10.1038/jcbfm.2015.14

Frackowiak, R. S., Herold, S., Petty, R. K., and Morgan-Hughes, J. A. (1988). The cerebral metabolism of glucose and oxygen measured with positron tomography in patients with mitochondrial diseases. Brain 111, 1009-1024. doi: 10.1093/brain/111.5.1009

Frackowiak, R. S., Lenzi, G. L., Jones, T., and Heather, J. D. (1980). Quantitative measurement of regional cerebral blood flow and oxygen metabolism in man using $15 \mathrm{O}$ and positron emission tomography: theory, procedure, and normal values. J. Comput. Assist. Tomogr. 4, 727-736. doi: 10.1097/00004728-198012000-00001
MG, and HC). RW was also supported by the Higher Education Funding Council for Wales. KM was supported by Wellcome grant 200804/Z/16/Z. TO was supported by the Royal Academy of Engineering and Wellcome Centre for Integrative Neuroimaging is supported by core funding from Wellcome (203139/Z/16/Z).

\section{ACKNOWLEDGMENTS}

We would like to thank Wellcome for supporting this work: Wellcome Strategic Award, Multi-scale and multi-modal assessment of coupling in the healthy and diseased brain, grant reference 104943/Z/14/Z.

\section{SUPPLEMENTARY MATERIAL}

The Supplementary Material for this article can be found online at: https://www.frontiersin.org/articles/10.3389/frai.2020. 00012/full\#supplementary-material

Supplementary Table 1 | Demographic data for healthy volunteers.

Frau-Pascual, A., Vincent, T., Sloboda, J., Ciuciu, P., and Forbes, F. (2014). "Physiologically informed bayesian analysis of ASL fMRI data," in Bayesian and Graphical Models for Biomedical Imaging. Lecture Notes in Computer Science, Vol. 8677 (Cham: Springer), 37-48. doi: 10.1007/978-3-319-12289-2_4

Friston, K. J., Holmes, A. P., Poline, J. B., Grasby, P. J., Williams, S. C. R., Frackowiak, R. S. J., et al. (1995). Analysis of fmri time-series revisited. Neuroimage 2, 45-53. doi: 10.1006/nimg.1995.1007

Gauthier, C. J., Desjardins-Crepeau, L., Madjar, C., Bherer, L., and Hoge, R. D. (2012). Absolute quantification of resting oxygen metabolism and metabolic reactivity during functional activation using QUO2 MRI. Neuroimage 63, 1353-1363. doi: 10.1016/j.neuroimage.2012.07.065

Gauthier, C. J., and Hoge, R. D. (2013). A generalized procedure for calibrated MRI incorporating hyperoxia and hypercapnia. Hum. Brain Mapp. 34, 1053-1069. doi: $10.1002 / \mathrm{hbm} .21495$

Germuska, M., Chandler, H. L., Stickland, R. C., Foster, C., Fasano, F., Okell, T. W., et al. (2019). Dual-calibrated fMRI measurement of absolute cerebral metabolic rate of oxygen consumption and effective oxygen diffusivity. Neuroimage 184, 717-728. doi: 10.1016/j.neuroimage.2018.09.035

Germuska, M., Merola, A., Murphy, K., Babic, A., Richmond, L., Khot, S., et al. (2016). A forward modelling approach for the estimation of oxygen extraction fraction by calibrated fMRI. Neuroimage 139, 313-323. doi: 10.1016/j.neuroimage.2016.06.004

Germuska, M., and Wise, R. G. (2019). Calibrated fMRI for mapping absolute $\mathrm{CMRO}_{2}$ : practicalities and prospects. Neuroimage 187, 145-153. doi: 10.1016/j.neuroimage.2018.03.068

Geurts, P., Ernst, D., and Wehenkel, L. (2006). Extremely randomized trees. Mach. Learn. 63, 3-42. doi: 10.1007/s10994-006-6226-1

Gjedde, A. (2002). Cerebral blood flow change in arterial hypoxemia is consistent with negligible oxygen tension in brain mitochondria. Neuroimage 17, 1876-1881. doi: 10.1006/nimg.2002.1272

Hayashi, T., Watabe, H., Kudomi, N., Kim, K. M., Enmi, J., Hayashida, K., et al. (2003). A theoretical model of oxygen delivery and metabolism for physiologic interpretation of quantitative cerebral blood flow and metabolic rate of oxygen. J. Cereb. Blood Flow. Metab. 23, 1314-1323. doi: 10.1097/01.WCB.0000090506.76664.00

Hertel, L., Phan, H., and Mertins, A. (2016). "Comparing time and frequency domain for audio event recognition using deep learning," in 2016 International Joint Conference on Neural Networks (IJCNN) (Vancouver, BC), 3407-3411. doi: 10.1109/IJCNN.2016.7727635

Hoge, R. D. (2012). Calibrated fMRI. Neuroimage 62, 930-937. doi: 10.1016/j.neuroimage.2012.02.022 
Hyder, F., Herman, P., Bailey, C. J., Moller, A., Globinsky, R., Fulbright, R. K., et al. (2016). Uniform distributions of glucose oxidation and oxygen extraction in gray matter of normal human brain: no evidence of regional differences of aerobic glycolysis. J. Cereb. Blood Flow. Metab. 36, 903-916. doi: 10.1177/0271678X15625349

Ibaraki, M., Shinohara, Y., Nakamura, K., Miura, S., Kinoshita, F., and Kinoshita, T. (2010). Interindividual variations of cerebral blood flow, oxygen delivery, and metabolism in relation to hemoglobin concentration measured by positron emission tomography in humans. J. Cereb. Blood Flow. Metab. 30, 1296-1305. doi: $10.1038 /$ jcbfm.2010.13

Ishii, K., Kitagaki, H., Kono, M., and Mori, E. (1996). Decreased medial temporal oxygen metabolism in Alzheimer's disease shown by PET. J. Nucl. Med. 37, 1159-1165.

Jenkinson, M., Beckmann, C. F., Behrens, T. E., Woolrich, M. W., and Smith, S. M. (2012). Fsl. Neuroimage 62, 782-790. doi: 10.1016/j.neuroimage.2011.09.015

Lajoie, I., Nugent, S., Debacker, C., Dyson, K., Tancredi, F. B., Badhwar, A., et al. (2017). Application of calibrated fMRI in Alzheimer's disease. Neuroimage Clin. 15, 348-358. doi: 10.1016/j.nicl.2017.05.009

Lebrun-Grandie, P., Baron, J. C., Soussaline, F., Loch'h, C., Sastre, J., and Bousser, M. G. (1983). Coupling between regional blood flow and oxygen utilization in the normal human brain. A study with positron tomography and oxygen 15 . Arch. Neurol. 40, 230-236. doi: 10.1001/archneur.1983.04050040060010

Leenders, K. L., Perani, D., Lammertsma, A. A., Heather, J. D., Buckingham, P., Healy, M. J., et al. (1990). Cerebral blood flow, blood volume and oxygen utilization. Normal values and effect of age. Brain 113, 27-47. doi: 10.1093/brain/113.1.27

Merola, A., Germuska, M. A., Murphy, K., and Wise, R. G. (2018). Assessing the repeatability of absolute $\mathrm{CMRO} 2, \mathrm{OEF}$ and haemodynamic measurements from calibrated fMRI. Neuroimage 173, 113-126. doi: 10.1016/j.neuroimage.2018.02.020

Merola, A., Germuska, M. A., Warnert, E. A., Richmond, L., Helme, D., Khot, S., et al. (2017). Mapping the pharmacological modulation of brain oxygen metabolism: the effects of caffeine on absolute $\mathrm{CMRO}_{2}$ measured using dual calibrated fMRI. Neuroimage 155, 331-343. doi: 10.1016/j.neuroimage.2017.03.028

Merola, A., Murphy, K., Stone, A. J., Germuska, M. A., Griffeth, V. E. M., Blockley, N. P., et al. (2016). Measurement of oxygen extraction fraction (OEF): an optimized BOLD signal model for use with hypercapnic and hyperoxic calibration. Neuroimage 129, 159-174. doi: 10.1016/j.neuroimage.2016.01.021

Mesejo, P., Saillet, S., David, O., Bénar, C., Warnking, J. M., and Forbes, F. (2015). "Estimating biophysical parameters from BOLD signals through evolutionarybased optimization," in Medical Image Computing and Computer-Assisted Intervention - MICCAI 2015, Lecture Notes in Computer Science, eds N. Navab, J. Hornegger, W. Wells, and A. Frangi (Cham: Springer), 9350, 528-535 doi: 10.1007/978-3-319-24571-3_63

Miles, K. A., and Williams, R. E. (2008). Warburg revisited: imaging tumour blood flow and metabolism. Cancer Imaging 8, 81-86. doi: 10.1102/1470-7330.2008.0011

Mintun, M. A., Lundstrom, B. N., Snyder, A. Z., Vlassenko, A. G., Shulman, G. L., and Raichle, M. E. (2001). Blood flow and oxygen delivery to human brain during functional activity: theoretical modeling and experimental data. Proc. Natl. Acad. Sci. U.S.A. 98, 6859-6864. doi: 10.1073/pnas.111164398

Morris, E. A., Juttukonda, M. R., Lee, C. A., Patel, N. J., Pruthi, S., Donahue, M. J., et al. (2018). Elevated brain oxygen extraction fraction in preterm newborns with anemia measured using noninvasive MRI. J. Perinatol. 38, 1636-1643. doi: 10.1038/s41372-018-0229-1

Pedregosa, F., Varoquaux, G., Gramfort, A., Michel, V., Thirion, B., Grisel, O., et al. (2011). Scikit-learn: Machine Learning in Python. J. Mach. Learn. Res. 12, 2825-2830. doi: 10.5555/1953048.2078195

Powers, W. J., Videen, T. O., Markham, J., Walter, V., and Perlmutter, J. S. (2011). Metabolic control of resting hemispheric cerebral blood flow is oxidative, not glycolytic. J. Cereb. Blood Flow. Metab. 31, 1223-1228. doi: $10.1038 / j \mathrm{cbfm} .2011 .5$

Reeve, H. W. J., and Brown, G. (2018). Diversity and degrees of freedom in regression ensembles. Neurocomputing 298, 55-68. doi: 10.1016/j.neucom.2017.12.066

Rosenfeld, A., and Kak, A. C. (1982). Digital Picture Processing. Vol. I \& II. New York, NY: Academic Press.

Ruijters, D., and Thevenaz, P. (2012). GPU Prefilter for accurate Cubic B-spline interpolation. Comput. J. 55, 15-20. doi: 10.1093/comjnl/bxq086

Safar, P. (1988). Resuscitation from clinical death: pathophysiologic limits and therapeutic potentials. Crit Care Med. 16, 923-941. doi: 10.1097/00003246-198810000-00003

Scheinberg, P., and Stead, E. A. (1949). The cerebral blood flow in male subjects as measured by the nitrous oxide technique. Normal values for blood flow, oxygen utilization, glucose utilization, and peripheral resistance, with observations on the effect of tilting and anxiety. J. Clin. Invest. 28, 1163-1171. doi: $10.1172 /$ JCI102150

Sollich, P., and Krogh, A. (1996). Learning with ensembles: how over-fitting can be useful. Adv. Neural Inform. Process. Syst. 8, 190-196.

Vafaee, M. S., and Gjedde, A. (2000). Model of blood-brain transfer of oxygen explains nonlinear flow-metabolism coupling during stimulation of visual cortex. J. Cereb. Blood Flow. Metab. 20, 747-754. doi: 10.1097/00004647-200004000-00012

Verweij, B. H., Amelink, G. J., and Muizelaar, J. P. (2007). Current concepts of cerebral oxygen transport and energy metabolism after severe traumatic brain injury. Prog. Brain. Res. 161, 111-124. doi: 10.1016/S0079-6123(06)61008-X

Wise, R. G., Harris, A. D., Stone, A. J., and Murphy, K. (2013). Measurement of OEF and absolute CMRO2: MRI-based methods using interleaved and combined hypercapnia and hyperoxia. Neuroimage 83, 135-147. doi: 10.1016/j.neuroimage.2013.06.008

Yue, J. B., Feng, H. K., Yang, G. J., and Li, Z. H. (2018). A comparison of regression techniques for estimation of above-ground winter wheat biomass using near-surface spectroscopy. Rem. Sens. 10:66. doi: 10.3390/rs10010066

Conflict of Interest: FF was employed by company Siemens Healthcare Ltd.

The remaining authors declare that the research was conducted in the absence of any commercial or financial relationships that could be construed as a potential conflict of interest.

Copyright (C) 2020 Germuska, Chandler, Okell, Fasano, Tomassini, Murphy and Wise. This is an open-access article distributed under the terms of the Creative Commons Attribution License (CC BY). The use, distribution or reproduction in other forums is permitted, provided the original author(s) and the copyright owner(s) are credited and that the original publication in this journal is cited, in accordance with accepted academic practice. No use, distribution or reproduction is permitted which does not comply with these terms. 